\title{
Prospective investigation of penile length with newborn male circumcision and second to fourth digit ratio
}

\author{
Jong Kwan Park, MD; ${ }^{\prime *}$ A. Ram Doo, MD, ${ }^{* *}$ Joo Heung Kim, MD, ${ }^{3}$ Hyung Sub Park, MD,4 Jung Mo Do, MD; ${ }^{5}$ \\ Hwang Choi, MD; ${ }^{5}$ Seung Chol Park, MD; ${ }^{6}$ Myung Ki Kim, MD; ${ }^{1+}$ Young Beom Jeong, MD; Hyung Jim Kim, MD; \\ Young Gon Kim, MD; Yu Seob Shin, MD't
}

\begin{abstract}
'Department of Urology, Chonbuk National University Medical School, Jeoniu, Korea; ${ }^{2}$ Department of Anesthesiology and Pain Medicine, Chonbuk National University Medical School, Jeonju, Korea; ${ }^{3}$ Department of Surgery, Severance Hospital, Yonsei University College of Medicine, Seoul, Korea; ${ }^{4}$ Department of Surgery, Seoul National University Bundang Hospital, Seoul National University College of Medicine, Gyeonggi, Korea; ${ }^{5}$ Department of Urology, Armed Forces Capital Hospital, Seongnam, Korea; 'Department of Urology, Wonkwang University School of Medicine and Hospital, Iksan, Korea
\end{abstract}

*Contributed equally to this study as co-first authors; ${ }^{\dagger}$ contributed equally to this study as co-corresponding authors.

Cite as: Can Urol Assoc J 2016;10(9-10):E296-9. http://dx.doi.org/10.5489/cuaj.3590

Published online September 13, 2016

\section{Abstract}

Introduction: We prospectively investigated the relationship between newborn male circumcision (NMC) and second to fourth digit ratio with penile length.

Methods: As participants for our study, we identified already circumcised young patients who visited our hospital for urological treatment. The age at which the circumcision had been done was assessed. The patients' height and weight were measured. Second to fourth digit ratio was calculated by measuring the second and fourth digit lengths. The flaccid and erectile penile lengths were measured from the base of the penis to the tip of the glans in standing position.

Results: A total of 248 patients were included in our study. In univariate analysis, height, second to fourth digit ratio, flaccid penile length, and age of circumcision were associated with erectile penile length. Among these variables, second to fourth digit ratio, flaccid penile length, and age of circumcision were significant predictive factors for erectile penile length in multivariate analysis. The subjects were divided into two groups, including 72 patients in the NMC group and 176 patients in the non-NMC group. No significant difference was found in height, weight, and second to fourth digit ratio between both groups. However, flaccid $(p<0.001)$ and erectile $(p=0.001)$ penile lengths were shorter in the NMC group than in the non-NMC group.

Conclusions: Despite the small number of subjects, this study shows that NMC was associated with shorter penile length. Second to fourth digit ratio, flaccid penile length, and age of circumcision were also significant predictive factors for erectile penile length. Further multicentre studies with larger number of subjects and biochemical analyses are needed for potential clinical applicability.

\section{Introduction}

Newborn male circumcision (NMC) is one of the oldest and most frequently performed operations. ${ }^{1}$ The recent survey of the American Academy of Pediatrics concluded that the health benefits of NMC outweigh the risks, and the benefits of NMC justify access to this procedure for those families who choose it. ${ }^{2}$ NMC offers numerous health benefits and protections against certain medical conditions, including human immunodeficiency virus, sexually transmitted infections, and penile cancer. ${ }^{3,4}$

While there is controversy about the best age for male circumcision, there are currently no studies that have investigated the association between penile length and the age at which circumcision was performed. The aim for this study is to figure out how NMC affects the growth of penis by investigating the association between penis size and the age of circumcision.

\section{Methods}

\section{Subjects}

As participants for our study, we identified men who were already circumcised among patients who visited our hospital for urological treatment from November 2014 to August 2015. Participants between the ages of 18 and 28 who had undergone circumcision previously were prospectively enrolled in this study. Men with hypospadias, urethral structure, Peyronie's disease, penile cancer, or a history of endocrine disease, urethroplasty, or other penile surgeries (except for circumcision) that may have a major influence on penile length were excluded. Study protocols and informed 
consent forms were approved by the Institutional Review Board (IRB; Number, AFMC-15065-IRB-15-059). Written informed consent was obtained from all subjects before study enrollment. This study was conducted in accordance with the good clinical practice and the international conference on harmonization guidelines, and in conformity with the ethical principles of the declaration of Helsinki. Patients were recruited prospectively and consecutively.

\section{Measurement}

All the patients' height and weight were measured. The age at which the circumcision had been done was assessed. The second and fourth digit lengths of the right hand and flaccid/erectile penile lengths were measured by a single investigator using a rigid ruler. The calculations were taken to the nearest $0.1 \mathrm{~cm}$. The second and fourth digit lengths of the right hand were measured directly on the ventral surfaces of the fingers, from the crease proximal to the palm at the base of each digit to the digit tip, and the second to fourth digit ratio was calculated. The flaccid penile length and erectile penile length were measured from the base of the penis (pubic bone) at the pubo-penile junction to the tip of the glans in standing position. The starting point was on the dorsal aspect of the penis at its base at the pubicpenile skin junction, pushing the pre-pubic fat pad against the pubic bone, and the tip of the penis was the other reference point. The penile erection was achieved by provision of adult magazine. The estimation was done swiftly in a warm private room to avoid any effect of temperature or touch on penile measurements.

\section{Statistical analysis}

Relationships between study variables were analyzed using Pearson's linear correlation. To identify the independent predictive factors influencing penile length, univariate and multivariate analyses were performed using linear regression models. Comparisons of data between Groups 1 (NMC) and 2 (non-NMC) were made using the two-tailed Student's t-test. Analysis was performed using SPSS 18.0 (SPSS Inc., Chicago, IL, U.S.), and differences were considered statistically significant when $\mathrm{p}$ values were less than 0.05 .

\section{Results}

A total of 248 patients were included in our study. Patients' characteristics are summarized in Table 1. Table 2 indicates the relationships between erectile penile length and other study variables. In univariate analysis using a linear regression model, height $(r=0.211 ; p=0.034)$, second to fourth digit ratio $(r=-0.205 ; p=0.007)$, flaccid penile length $(r=0.188$; $p<0.001)$, and age of circumcision $(r=-0.235 ; p=0.001)$ were associated with erectile penile length (Table 2). Among these variables, second to fourth digit ratio, flaccid penile length $(r=0.176 ; p<0.001)$, and age of circumcision $(r=0.216$; $p=0.001$ ) were significant predictive factors for erectile penile length in multivariate analysis using a linear regression model (Table 2).

The subjects were divided into two groups: the NMC group and the non-NMC group. The NMC group included 72 patients $(29.0 \%)$ who were circumcised during the newborn period; the non-NMC group included 176 patients $(71.0 \%)$ who were circumcised after the newborn period. There was no significant difference in height, weight, and second to fourth digit ratio between both groups (Table 3). However, flaccid $(p<0.001)$ and erectile $(p=0.001)$ penile lengths of the NMC group were shorter than those of the non-NMC group (Table 3 ).

\section{Discussion}

The major finding of our study is that second to fourth digit ratio and the age of circumcision were significant predictive factors for erectile penile length. Furthermore, the penile lengths of the NMC group were shorter than those of the non-NMC group. To our knowledge, this is the first study that focuses on association between penile length and NMC. Although, NMC offers numerous health benefits and protections against certain medical conditions, ${ }^{3,4}$ our results provide evidence suggesting that NMC may have a significant effect on penile length.

Androgen with androgen receptors are known to be necessary for the development of the penis. ${ }^{5,6}$ The difference of penile lengths between individuals seems to be highly influenced by prenatal testosterone. ${ }^{7-9}$ Like digit development, penile growth is influenced by prenatal testosterone. Choi et al reported that the second to fourth digit ratio was a significant predictive factor for stretched penile length $(r=-0.201 ; p=0.024)$ in multivariate analysis using a linear regression model. ${ }^{10}$ Stretched penile length was found to be negatively associated with digit ratio. ${ }^{10}$ The effects of

\begin{tabular}{lcc}
\hline \multicolumn{3}{l}{ Table 1. Clinical characteristics of the study population } \\
\hline Variables & Mean \pm SD & Median (range) \\
\hline Age (year) & $22.69 \pm 1.75$ & $22.0(20-29)$ \\
Height $(\mathrm{cm})$ & $175.48 \pm 4.94$ & $175.0(163-186)$ \\
Weight $(\mathrm{kg})$ & $71.85 \pm 8.69$ & $71.0(50-100)$ \\
Second digit length $(\mathrm{cm})$ & $8.01 \pm 0.77$ & $7.9(6.9-10.5)$ \\
Fourth digit length $(\mathrm{cm})$ & $8.18 \pm 0.88$ & $8.0(5.5-10.7)$ \\
Digit ratio & $0.98 \pm 0.08$ & $0.97(0.84-1.49)$ \\
Flaccid penile length $(\mathrm{cm})$ & $7.39 \pm 1.52$ & $7.3(4.8-11.0)$ \\
Erectile penile length $(\mathrm{cm})$ & $13.53 \pm 1.68$ & $13.1(10.1,18.5)$ \\
Age of circumcision & $9.69 \pm 5.91$ & $11.0(0-19)$ \\
\hline Digit ratio: second digit length/fourth digit length. SD: standard deviation.
\end{tabular}

Digit ratio: second digit length/fourth digit length. SD: standard deviation. 


\begin{tabular}{lcccc}
\hline $\begin{array}{l}\text { Table 2. Relationships between erectile penile length and } \\
\text { other study variables }\end{array}$ & \multicolumn{4}{c}{ Erectile penile length } \\
\hline & \multicolumn{3}{c}{ Univariate } & Multivariate \\
\cline { 2 - 5 } & $\mathbf{r}$ p value & $\mathbf{r}$ & p value \\
\hline Age & 0.052 & 0.528 & & \\
Height & 0.211 & 0.034 & 0.196 & 0.064 \\
Weight & -0.029 & 0.698 & & \\
Second digit length & -0.047 & 0.567 & & \\
Fourth digit length & 0.065 & 0.341 & & \\
Digit ratio & -0.205 & 0.007 & -0.198 & 0.009 \\
Flaccid penile length & 0.188 & $<0.001$ & 0.176 & $<0.001$ \\
Age of circumcision & 0.235 & $<0.001$ & 0.216 & 0.001 \\
\hline
\end{tabular}

prenatal testosterone may, in part, explain the differences in adult penile length. The results of our study are similar to the existing findings. Androgens and a functioning androgen receptor are known to be necessary for normal development of the human penis. ${ }^{11}$ The ratio of second to fourth digit length is sexually dimorphic in humans and is thought to be fixed early in development. ${ }^{11}$ During the fetal period, high concentrations of testosterone lead to high testicular activity, resulting in a lower digit ratio. In our study, men with a lower second to fourth digit ratio tended to have a longer penile length.

A large observational cohort study demonstrated that complications of NMC occurred in eight out of 476 boys. ${ }^{12}$ Excessive bleeding occurred in three patients in this study and was the most common complication. ${ }^{12}$ Although the numbers were small, from a mechanistic point of view, bleeding can lead to damage of the normal structures, which in turn, can affect penile length. NMC can also remove the loose flap of skin that covers and protects the rounded top part of the penis that contains nerves, blood vessels, and a tiny part of muscle, which can lead to shorter penile length.

Several studies showed that normal flaccid penile length ranges between 7.6 and $13.0 \mathrm{~cm}$, and erectile penile length ranges between 12.7 and $17.7 \mathrm{~cm} .{ }^{13,14}$ The results of our study were similar to the existing findings. The flaccid penile length was $7.39 \mathrm{~cm}$ and erectile penile length was 13.53 $\mathrm{cm}$. The penile length was a concern for $68.3 \%$ of 200 men in one study. ${ }^{15}$ Men and women agreed that the ideal penis length was longer than what they thought was average. ${ }^{16}$ Concerns about penile length affect men's sexual satisfaction and functioning. A smaller penis decreases sexual confidence, which may be why penis size is related to sexual function. ${ }^{17}$ Thus, penile length has a significant role in men's sexual satisfaction and our results suggest that NMC could be reconsidered in terms of penile length.

The small size of subjects and the absence of biochemical markers, such as testosterone levels, can be limitations of this analysis. Further multicentre studies including larger number

\begin{tabular}{lccc}
\hline $\begin{array}{l}\text { Table 3. Comparison of study variables between NMC vs. } \\
\text { non-NMC groups }\end{array}$ & \multicolumn{3}{c}{} \\
\hline & NMC (n=72) & $\begin{array}{c}\text { Non-NMC } \\
(\mathbf{n = 1 7 6 )}\end{array}$ & p value \\
\hline Age (years) & $22.33 \pm 0.88$ & $22.84 \pm 1.99$ & 0.847 \\
Height (cm) & $175.63 \pm 4.90$ & $175.49 \pm 4.91$ & 0.642 \\
Second digit length (cm) & $7.96 \pm 0.69$ & $8.03 \pm 0.80$ & 0.507 \\
Fourth digit length (cm) & $8.13 \pm 0.70$ & $8.20 \pm 0.95$ & 0.593 \\
Digit ratio & $0.98 \pm 0.6$ & $0.98 \pm 0.09$ & 0.717 \\
Flaccid penile length (cm) & $6.78 \pm 1.43$ & $7.65 \pm 1.56$ & $<0.001$ \\
Erectile penile length (cm) & $12.97 \pm 1.58$ & $13.75 \pm 1.67$ & 0.001 \\
\hline $\begin{array}{l}\text { Digit ratio: second digit length/fourth digit length; values are shown as mean } \pm \text { standard } \\
\text { deviation. NMC: newborn male circumcision. }\end{array}$
\end{tabular}

of subjects and blood sampling to evaluate sex hormones are needed in the near future.

\section{Conclusion}

In conclusion, second to fourth digit ratio, flaccid penile length, and age of circumcision were significant predictive factors for erectile penile length. Furthermore, the penile lengths of the NMC group were shorter than those of the non-NMC group. Because there are no set guidelines for the optimal age at which to perform circumcision, physicians will be continually faced with the issue of when to perform the procedure. This information will be helpful for providing more professional counselling to parents with a newborn boy.

Competing interests: The authors report no competing personal or financial interests.

This paper has been peer-reviewed.

\section{References}

1. Kaplan GW. Complication of circumcision. Urol Clin North Am 1983;10:543-9.

2. American Academy of Pediatrics Task Force on Circumcision. Circumcision policy statement. Pediatrics 2012;130:0756-85.

3. Gray RH, Kigozi G, Serwadda D, et al. Male circumcision for HIV prevention in men in Rakai, Uganda: A randomized trial. Lancet 2007;369:657-66. http://dx.doi.org/10.1016/S0140-6736(07)60313-4

4. Weiss HA. Male circumcision as a preventive measure against HIV and other sexually transmitted diseases. Curr Opin Infect Dis 2007;20:66-72. http://dx.doi.org/10.1097/QC0.0b013e32801 lab73

5. Quigley CA, de Bellis A, Marschke KB, et al. Androgen receptor defects: Historical, clinical, and molecular perspectives. Endocr Rev 1995;16:271-321. http://dx.doi.org/10.1210/edr-16-3-271

6. Byne W. Developmental endocrine influences on gender identity: Implications for management of disorders of sex development. Mt Sinai J Med 2006;73: 950-9.

7. Baskin $L S$, Sutherland $R S$, DiSandro $M J$, et al. The effect of testosterone on androgen receptors and human penile growth. J Urol 1997;158:1113-8. http://dx.doi.org/10.1016/S0022-5347(01)64400-8

8. Boas $M$, Boisen $K A$, Virtanen $H E$, et al. Postnatal penile length and growth rate correlate to serum testosterone levels: A longitudinal study of 1962 normal boys. Eur J Endocrinol 2006;154:125-9. http:// dx.doi.org/10.1530/eje.1.02066

9. Camurdan $A D, O z M O$, Ithan $M N$, et al. Current stretched penile length: Cross-sectional study of 1040 healthy Turkish children aged 0-5 years. Urology 2007;70:572-5. http://dx.doi.org/10.1016/i. urology.2007.04.051

10. Choi IH, Kim KH, Jung H, et al. Second to fourth digit ratio: A predictor of adult penile length. Asian J Androl 2011;13:710-4. http://dx.doi.org/10.1038/aja.2011.75 
11. Kondo T, Zakany J, Innis JW, et al. Of fingers, toes, and penises. Nature 1997;390:29. http://dx.doi. org $/ 10.1038 / 36234$

12. Wiswell TE, Tencer HL, Welch CA, et al. Circumcision in children beyond the neonatal period. Pediatrics 1993:92:791-3.

13. Wessells H, Lue TF, McAninch JW. Penile length in the flaccid and erect states: Guidelines for penile augmentation. J Urol 1996;156:995-7. hittp://dx.doi.org/10.1016/50022-5347(01)65682-9

14. Vardi Y, Har-Shai Y, Gil T, et al. A critical analysis of penile enhancement procedures for patients with normal penile size: surgical techniques, success, and complications. Eur Urol 2008;54:1042-50. http:// dx.doi.org/10.1016/j.eururo.2008.07.080

15. Tiggemann M, Martins Y, Churchett L. Beyond muscles: Unexplored parts of men's body image. J Health Psychol 2008;13:1163-72. http://dx.doi.org/10.1177/1359105308095971
16. Johnston L, McLellan T, McKinlay A. (Perceived) Size really does matter: Male dissatisfaction with penis size. Psychology of Men and Masculinity 2014;15:225-8. http://dx.doi.org/10.1037/a0033264

17. Althof SE, Cappelleri IC, Shpilsky A, et al. Treatment responsiveness of the self-esteem and relationship questionnaire in erectile dysfunction. Urology 2003;61:888-92. http://dx.doi.org/10.1016/500904295(03)00041-4

Correspondence: Dr. Yu Seob Shin, Department of Urology, Chonbuk National University Medical School, Jeoniu, Korea; ball1210@hanmail.net 